\title{
A Case Report of Vascular Ectasias of GI Tract Presenting as Occult Gastro-Intestinal Bleed Diagnosed on Capsule Endoscopy
}

\author{
Santosh Hajare ${ }^{1}$, Suhas. M², J.N.M.C; Belgaum
}

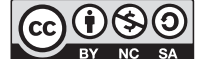

DOI: https://doi.org//0.3329/jom.v2 Ii2.502/8

Copyright: (C) 2020 Hahare $S$ et al. This is an open access article published under the Creative Commons Attribution-NonCommercial-NoDerivatives 4.0 International License, which permits use, distribution and reproduction in any medium, provided the original work is properly cited, is not changed in any way and it is not used for commercial purposes.
\end{abstract}

Received: 19 December 2020;

Accepted: 03 September 2020

\section{Introduction :}

Obscure GI bleed is a condition when there is definitive Gastro-Intestinal(GI) bleed, whose source remains elusive despite an initial negative Upper-GI endoscopy and a colonoscopy ${ }^{1}$. Occult GI bleed can occur anywhere in the GI tract. But the diagnosis becomes extremely tricky when endoscopic reports are negative for gross bleeds. The newer diagnostic modalities like Capsule Endoscopy (CE ) thus perfectly fit into this vacuum of diagnostic modalities. We report one such case diagnosed by Capsule Endoscopy.

\section{Case Report :}

A 52 year old otherwise healthy female patient presented to the casualty with complaints of generalized weakness \& easy fatigability since 1 month. There was no history of any bleeding tendencies. Routine investigations showed a severe anemia of $6.7 \mathrm{~g} / \mathrm{dl}$ Hemoglobin. Stool for occult blood was positive. This prompted us to focus on the GI tract for the source of the bleeder. She later underwent an upper GIendoscopy which was normal. A colonoscopy also showed a normal study. Then suspecting the source of the bleed to be between the duodenum $3^{\text {rd }}$ part and the ileo-caecal junction, the patient was subjected for a capsule endoscopy (CE) examination. This showed multiple bleeding spots in the jejunum which were actively bleeding. The patient was then posted for surgery, when she underwent a short segment resection anastomosis of the jejunum.

On histopathological examination of the resected sample, it showed congested mucosa with lamina propria showing numerous ectatic blood vessels of varying sizes suggestive of vascular ectasias.

1. Professor, Department of Gastroenterology, Jawaharlal Nehru Medical College, Kle University.

2. Assistant Professor, Department of Neurology ,Bangalore Medical College \& Research Institute

Correspondence: Assistant Professor, Department of Neurology, Bangalore Medical College \& Research Institute, Phone number: +91-9900284936, E-mail: suhas.murali@gmail.com

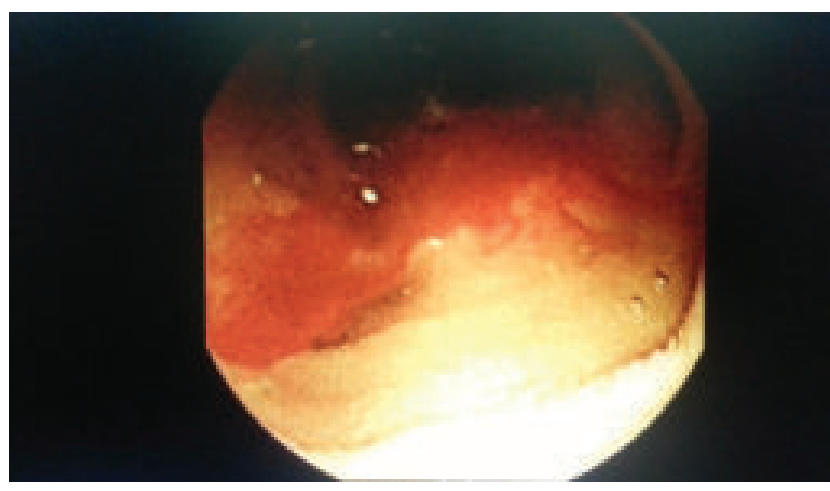

Fig.-1 : The picture shows the capsule endoscopy showing active bleeding from the jejunum.

\section{Results :}

Post-operatively the patient showed remarkable improvement with no sequele and was discharged 10 days later from hospital.

\section{Discussion :}

Gastro-Intestinal bleeds that occur as a result of bleeders in between the Ligament of Treitz and the Ileo-Caecal valve; in other words between that part of the Gastro-intestinal tract not visualized by the routine Upper-GI scopy and colonoscopy accounts for approximately $5 \%$ of gastrointestinal bleeding ${ }^{2}$. Amongst the most common causes bleeding from this region includes angiectasias which accounts for $30-40 \%$ amongst these; specially in elderly ${ }^{3}$. Other important causes of occult GI bleeds from the small bowel includes NSAID induced enteropathy, IBD with ulcers or erosions, tumours, radiation enteropathy, Dieulafoy's lesions \& small bowel varices. The location of these lesions make it extremely tricky to diagnose.

The capsule endoscope however lets us have a visualization of the entire small intestine.

Compared to intraoperative enteroscopy, Capsule Endoscopy had a sensitivity of $95 \%$ and specificity of $75 \%$ in a prospective of 47 patients in. ${ }^{4}$ 
Comapredto CT angiography and standard angiography, Capsule Endoscopy detected more bleeding source lesions (72\% with VCEvs $24 \%$ with CTA and $56 \%$ with angiography). ${ }^{5}$

Benefits of capsule endoscopy include its non-invasive nature, better patient compliance and safety.

But drawbacks include inability as a therapeutic intervention, many false positive results and chances of erratic passage causing missed lesions.

If significant lesions like the one discovered in our patient is detected, the patient is referred for specific management of the cause. If the initial capsule endoscopy is negative one more attempt maybe done. If the second capsule endoscopy is also negative then the patient maybe managed conservatively.

\section{Conclusion :}

The main objective of this case report was to highlight the role of capsule endoscopy in reaching and identifying occult bleeding points which were till recently inaccessible. Even though capsule endoscopy lacks the therapeutic intervention needed, it can direct the physician on further course of management.

Hence we suggest that Capsule endoscopy as the first investigation of choice for evaluation of small bowel in patients with occult gastro-intestinal bleeds.

\section{References :}

1. Thomas J.S, Dennis M.J. Sleisenger and Fordtran's Gastrointestinal and Liver Diseases $9^{\text {th }}, 19: 285$

2. Katz LB. The role of surgery in occult gastrointestinal bleeding. SeminGastrointest Dis 1999;10:78-81.

3. Foutch PG. Angiodysplasia of the gastrointestinal tract. Am J Gastroenterol 1993;88:807-18.

4. Hartmann D, Schmidt H, Bolz G, et al. A prospective twocenter study comparing wireless capsule endoscopy with intraoperative enteroscopy in patients with obscure GI bleeding. GastrointestEndosc 2005; 61:826-32.

5. Saperas E, Dot J, Videla S, et al. Capsule endoscopy versus computed tomographic or standard angiography for the diagnosis of obscure gastrointestinal bleeding. Am J Gastroenterol 2007;102:731-7. 